\title{
CONDIÇÕES MICROMETEOROLÓGICAS ENTRE UMA ÁREA DE FLORESTA E A CAMPO
}

\author{
Bruna Matos Salvador ${ }^{1}$, Rosandro Boligon Minuzzi²
}

\begin{abstract}
RESUMO - O objetivo deste trabalho foi comparar a variação da temperatura do ar, umidade relativa do ar (UR) e temperatura no ponto de orvalho (Td) durante o inverno e verão, em diferentes escalas temporais (diário, diurno e noturno), numa área de floresta e a campo, no município de Águas Mornas, em Santa Catarina. A correlação das variáveis entre os ambientes foi analisada com o uso da regressão linear, onde considerou-se os dados obtidos a campo como a variável independe $\mathrm{X}$ e as obtidas na floresta como variável dependente Y. A significância estatística das regressões foi testada com o uso do valor-p. As diferenças entre as médias e variâncias dos dados entre os ambientes, épocas do ano e escala temporal, foram testadas ao nível de 5\% de significância usando os testes $\mathrm{t}$ de Student e F de Snedecor, respectivamente. Todas as análises estatísticas foram realizadas com o uso do software Past 3.0. Observou-se diferença entre os dois ambientes da temperatura média e máxima do ar e umidade relativa durante o verão. A temperatura do ar e do ponto de orvalho entre os ambientes apresentaram as maiores correlações. Assim, pode-se estimar estes elementos climáticos para floresta, a partir de dados obtidos a campo.
\end{abstract}

Palavras chave: microclima, temperatura do ar, temperatura do ponto de orvalho, umidade relativa.

\section{MICROMETEOROLOGICAL CONDITIONS BETWEEN AN AREA OF FOREST AND THE FIELD}

\begin{abstract}
The objective of this work was to compare the variation of air temperature, relative air humidity (RH) and dew point temperature (Td) during winter and summer, at different time scales (daily, daytime and nightly) in a area of forest and field, in the Águas Mornas City, in Santa Catarina. The correlation of the variables between the environments was analyzed with the use of Linear Regression, where the data obtained in the field were considered as the independent variable $X$ and those obtained in the forest as dependent variable $Y$. The statistical significance of the regressions was tested with the use of the p-value. The differences between means and variances of data between environments, times of the year and time scale were tested at the 5\% level of significance using Student's t test and Snedecor's F test, respectively. All statistical analyzes were performed using the Past 3.0 software. It was observed a difference between the two environments of the average and maximum air temperature and relative humidity during the summer. The air temperature and the dew point between the environments presented the highest correlations. Thus, we can estimate these climatic elements for forest, from data obtained in the field.
\end{abstract}

Keywords: air temperature, dew point temperature, microclimate, relative humidity.

Agrônoma na empresa Cooperativa Agrícola Dom Fruto, BR-282, km 208, Índios, 88533-000, Lages, SC. brumsalvador@gmail.com

2 Professor do Departamento de Engenharia Rural, Universidade Federal de Santa Catarina, Av. Admar Gonzaga, 1346, Itacorubi, 88034001, Florianópolis, SC. rosandro.minuzzi@ufsc.br 


\section{INTRODUÇÃO}

O desenvolvimento de pesquisas na área da climatologia geográfica nas diferentes escalas do clima são fundamentais para a compreensão da interação entre os elementos climáticos e o meio natural ou antropizado, necessitando desta forma, a coleta de dados em pontos específicos para entender as particularidades do microclima em cada ambiente (Lima, 2014).

Zheng et al. (2000) destacam que o clima é considerado um dos fatores ambientais mais importantes, uma vez que ele é capaz de afetar a estrutura e funcionamento dos ecossistemas. Assim, a importância no estudo da variação microclimática em diferentes estruturas florestais reside no entendimento de que, os fatores que envolvem o microclima e suas ações ao longo do tempo podem fornecer conhecimento em relação a fenômenos ecológicos e o desenvolvimento de espécies e indivíduos (Salim Neto, 2017).

O interior de uma floresta tem um funcionamento complexo e dinâmico com a atuação de um microclima que difere em função das características da vegetação e de seu grau de conservação (Silva, 2016). O microclima de uma floresta é descrita pela alteração na luz que penetra através da cobertura das árvores. Nas áreas com árvores tipo coníferas a luz é fortemente reduzida, mas pouco modificada qualitativamente. Já nas áreas com árvores tipo folhosas, a luz sofre uma grande absorção seletiva apresentando uma tonalidade amarelo esverdeada quando as árvores estão com 11 folhas. Numa floresta temperada, a iluminação ao nível do solo, pode descer a $2 \%$ da iluminação de um terreno descoberto. Porém na floresta tropical, a iluminação varia entre 0,1 e $1 \%$ (Dajoz, 1978). Mais recentemente, Hernandes et al. (2004) mostraram que a radiação solar transmitida para o interior de uma floresta semidecídua foi em média 5,5\% da radiação solar medida em área externa, variando de $3,3 \%$ no verão a $8,2 \%$ no inverno.

Para Vourlitis e Rocha (2010) a mudança na cobertura vegetal, como a redução nas áreas de floresta, pode ter consequências sobre o clima local e apresentar alterações no funcionamento do ecossistema. Isso ocorre pelo fato de cada tipo de cobertura possuir diferentes propriedades físicas e trocas biogeoquímicas (Bonan, 2009).

A cobertura vegetal além de bloquear incidência de radiação, é capaz de absorver parte dela e contribuir para o equilíbrio do balanço das energias (Hernandes, 2002). Oke (1978) afirma que a vegetação arbórea deve proporcionar sombra e auxiliar na diminuição da temperatura, a partir do consumo de calor latente por evaporação.
Baseado nas simulações de modelos climáticos, as florestas tropicais mantêm altas taxas de evapotranspiração, menor temperatura do ar e maior volume de água precipitada que áreas de pastagens (Fisch, 1996; Alves et al., 1999; Betts et al., 2008). Geiger et al. (2012) especificam que os microclimas nas florestas são criadas devido as suas características fisiográficas, estrutura e composição, resultando em ambiente com menor velocidade do vento, mais úmido e com menor perda e/ou ganho de radiação. Portanto, a compreensão e a modelagem do microclima de floresta são necessárias para entender a variação espacial e temporal nos processos biológicos. No âmbito das mudanças climáticas, esse conhecimento ajudará a identificar estratégias eficientes para adaptar o manejo florestal a importantes objetivos sociais, como a produção de madeira, o sequestro de carbono e a conservação da biodiversidade (Greiser et al., 2018).

Por isso o presente trabalho tem como objetivo comparar a variação microclimática durante o inverno e verão, em diferentes períodos do dia, entre uma área de floresta e a campo no município de Águas Mornas, em Santa Catarina.

\section{MATERIAL E MÉTODOS}

A coleta de dados foi realizada durante o período de julho à agosto de 2017 e dezembro à fevereiro de 2018, no distrito do Rio do Cedro, pertencente ao município de Águas Mornas (latitude $27,75^{\circ}$ sul e longitude $48,96^{\circ}$ oeste e altitude de 371 metros), numa área limítrofe ao Parque Estadual da Serra do Tabuleiro. Foram obtidos a cada 30 minutos, três variáveis climáticas (umidade relativa do ar (UR), temperatura do ar $\left({ }^{\circ} \mathrm{C}\right)$ e temperatura do ponto de orvalho (Td)) para dois ambientes distintos, a saber: um de vegetação rasteira, com gramíneas, sendo representativo das condições padrões para a instalação de uma estação meteorológica (WMO, 2013), e o outro, no interior de uma floresta ombrófila densa composta por árvores de diferentes alturas em terreno com pequena inclinação, estando distantes entre si, cerca de 270 metros.

Conforme metodologia de Köppen-Geiger, o clima na região é classificado como Cfb, isto é, mesotérmico com grande variação de temperatura durante o ano que varia de $12,8^{\circ} \mathrm{C}$ (julho) a $21,7^{\circ} \mathrm{C}$ (janeiro) e pluviosidade média bem distribuída ao ano que totaliza $1691 \mathrm{~mm}$ (Alvares et al., 2013).

O período de obtenção dos dados ocorreu do dia 09/07/2017 a 04/08/2017, sendo representativo do inverno e do dia 01/12/2017 a 16/02/2018, representando 
o verão, onde os termohigrógafos ficaram instalados no interior de um abrigo meteorológico a uma altura de 1,5 m. A temperatura do ponto de orvalho foi estimada pelo instrumento.

As comparações das variáveis climáticas para o inverno e verão, foram feitas em diferentes escalas de tempo, sendo na diária e nos períodos diurno ( 7 às $17 \mathrm{~h}$ e 6 às 19h, para o inverno e verão, respectivamente) e noturno (17 às $7 \mathrm{~h}$ e 19 às $6 \mathrm{~h}$, para o inverno e verão, respectivamente). As análises das temperaturas mínima e máxima do ar foram restritas apenas na escala diária.

A sincronia das variáveis entre os ambientes foi analisada com o uso da regressão linear, onde considerouse os dados obtidos a campo como a variável independente $\mathrm{X}$ e as obtidas na floresta como variável dependente Y. A significância estatística das regressões foi testada com o uso do valor-p. As diferenças entre as médias e variâncias dos dados entre os ambientes, épocas do ano e escala temporal, foram testadas ao nível de $5 \%$ de significância usando os testes $\mathrm{t}$ de Student e F de Snedecor, respectivamente. Todas as análises estatísticas foram realizadas com o uso do software Past 3.0 (Hammer et al., 2001).

\section{RESULTADOS E DISCUSSÃO}

Na Tabela 1 estão apresentadas as médias e variâncias obtidas a campo e a floresta, em diferentes escalas temporais para o verão e inverno, da temperatura média (1a), temperatura mínima (1b) e máxima do ar (1c), umidade relativa (UR) (1d) e temperatura do ponto de orvalho (Td) (1e).

As diferenças estatisticamente significativas somente foram observadas no verão e para a UR e temperaturas média e máxima do ar. Apenas para a UR essas diferenças foram observadas nas três escalas de tempo analisadas, variando de $5 \%$ a $14,2 \%$ para o período noturno e diurno, respectivamente (Tabela 1d). Para a temperatura média, as diferenças entre os ambientes ficaram restritas a escala diária, provavelmente em resposta ao período diurno, em que, a temperatura do ar a campo esteve em média, $2,45^{\circ} \mathrm{C}$ acima a da registrada na floresta (Tabela 1a). Mais expressivo, foi a diferença da temperatura máxima no verão que foi em média $3,14^{\circ} \mathrm{C}$ entre os ambientes (Tabela 1c).

Em suma, durante o verão a temperatura do ar/ UR a campo são maiores/menores do que numa floresta densa, principalmente, durante o dia. Biudes et al. (2012) atestam esses resultados, quando ao avaliarem a mudança no microclima de área de floresta para pastagem, encontraram diminuição de $5,7 \%$ na UR e aumento de $6,6 \%$ na temperatura máxima do ar. Lin (2007) acrescenta que a sombra projetada pelas árvores podem afetar significativamente o microclima na área, consequentemente, afetando a demanda evaporativa no solo.

Tabela 1 - Média e variância ${ }^{1}$ da temperatura média (1a), temperatura mínima (1b), temperatura máxima do ar (1c), umidade relativa do ar (1d) e temperatura do ponto de orvalho (1d) nos ambientes a campo e a floresta, durante o inverno e verão em diferentes escalas do tempo.

\begin{tabular}{|c|c|c|c|c|}
\hline \multirow[b]{3}{*}{$1 \mathrm{a}$} & \multicolumn{4}{|c|}{ Temperatura média do $\operatorname{ar}\left({ }^{\circ} \mathrm{C}\right)$} \\
\hline & \multicolumn{2}{|c|}{ Média } & \multicolumn{2}{|c|}{ Variância } \\
\hline & Campo & Floresta & Campo & Floresta \\
\hline Inverno diário & $17,49 \mathrm{~A}$ & $17,63 \mathrm{~A}$ & $9,19 \mathrm{~A}$ & $9,17 \mathrm{~A}$ \\
\hline Inverno noturno & $16,87 \mathrm{~A}$ & $17,01 \mathrm{~A}$ & $12,55 \mathrm{~A}$ & $12,57 \mathrm{~A}$ \\
\hline Inverno diurno & $18,37 \mathrm{~A}$ & $18,48 \mathrm{~A}$ & $7,14 \mathrm{~A}$ & $6,97 \mathrm{~A}$ \\
\hline Verão diário & $22,04 \mathrm{~A}$ & $20,61 \mathrm{~B}$ & $2,70 \mathrm{~A}$ & $2,27 \mathrm{~A}$ \\
\hline Verão noturno & $19,88 \mathrm{~A}$ & $19,60 \mathrm{~A}$ & $2,66 \mathrm{~A}$ & $2,14 \mathrm{~A}$ \\
\hline Verão diurno & $23,86 \mathrm{~A}$ & $21,41 \mathrm{~B}$ & $4,75 \mathrm{~A}$ & $3,28 \mathrm{~A}$ \\
\hline $1 \mathrm{~b}$ & \multicolumn{4}{|c|}{ Temperatura mínima do ar $\left({ }^{\circ} \mathrm{C}\right)$} \\
\hline Inverno & $10,08 \mathrm{~A}$ & $10,96 \mathrm{~A}$ & $10,92 \mathrm{~A}$ & $15,61 \mathrm{~A}$ \\
\hline Verão & $18,09 \mathrm{~A}$ & $17,83 \mathrm{~A}$ & $3,72 \mathrm{~A}$ & $2,88 \mathrm{~A}$ \\
\hline 1c & \multicolumn{4}{|c|}{ Temperatura máxima do ar $\left({ }^{\circ} \mathrm{C}\right)$} \\
\hline Inverno & $23,47 \mathrm{~A}$ & $22,14 \mathrm{~A}$ & $15,99 \mathrm{~A}$ & $15,06 \mathrm{~A}$ \\
\hline Verão & $27,55 \mathrm{~A}$ & $24,41 \mathrm{~B}$ & $11,69 \mathrm{~A}$ & $8,04 \mathrm{~A}$ \\
\hline $1 \mathrm{~d}$ & \multicolumn{4}{|c|}{ Umidade relativa do ar (\%) } \\
\hline Inverno diário & $43,74 \mathrm{~A}$ & $45,59 \mathrm{~A}$ & $21,98 \mathrm{~A}$ & $26,98 \mathrm{~A}$ \\
\hline Inverno noturno & $46,22 \mathrm{~A}$ & $47,17 \mathrm{~A}$ & $7,70 \mathrm{~A}$ & $9,01 \mathrm{~A}$ \\
\hline Inverno diurno & $40,29 \mathrm{~A}$ & $40,30 \mathrm{~A}$ & $67,82 \mathrm{~A}$ & $76,59 \mathrm{~A}$ \\
\hline Verão diário & $85,16 \mathrm{~A}$ & $94,90 \mathrm{~B}$ & $38,58 \mathrm{~A}$ & $23,97 \mathrm{~B}$ \\
\hline Verão noturno & $91,61 \mathrm{~A}$ & $96,60 \mathrm{~B}$ & $24,33 \mathrm{~A}$ & $14,58 \mathrm{~B}$ \\
\hline Verão diurno & $79,13 \mathrm{~A}$ & $93,37 \mathrm{~B}$ & $87,35 \mathrm{~A}$ & $41,01 \mathrm{~B}$ \\
\hline $1 \mathrm{e}$ & \multicolumn{4}{|c|}{ Temperatura do ponto de orvalho $\left({ }^{\circ} \mathrm{C}\right)$} \\
\hline Inverno diário & $16,22 \mathrm{~A}$ & $16,77 \mathrm{~A}$ & $17,39 \mathrm{~A}$ & $13,07 \mathrm{~A}$ \\
\hline Inverno noturno & $16,17 \mathrm{~A}$ & $16,50 \mathrm{~A}$ & $17,99 \mathrm{~A}$ & $15,78 \mathrm{~A}$ \\
\hline Inverno diurno & $16,29 \mathrm{~A}$ & $17,16 \mathrm{~A}$ & $16,85 \mathrm{~A}$ & $9,91 \mathrm{~B}$ \\
\hline Verão diário & $19,17 \mathrm{~A}$ & $19,7 \mathrm{~A}$ & $3,08 \mathrm{~A}$ & $2,58 \mathrm{~A}$ \\
\hline Verão noturno & $18,78 \mathrm{~A}$ & $19,05 \mathrm{~A}$ & $2,78 \mathrm{~A}$ & $2,69 \mathrm{~A}$ \\
\hline Verão diurno & $19,69 \mathrm{~A}$ & $20,24 \mathrm{~A}$ & $3,65 \mathrm{~A}$ & $3,04 \mathrm{~A}$ \\
\hline
\end{tabular}

${ }^{1}$ Letras iguais entre as médias e variâncias na mesma linha não diferem estatisticamente entre si pelo teste t e teste $\mathrm{F}$, respectivamente, ao nível de $5 \%$ de significância. 
Baker et al. (2014), afirmam que o microclima dentro de uma floresta geralmente tende a ser estável, não apresentando grandes variações ao longo do tempo, pelo fato do dossel provocar efeito regulador no balanço de energia, tornando relativamente estável a temperatura e a umidade. Entretanto, quando ocorrem modificações naturais ou provocadas pelo homem alteram a estrutura da floresta, possibilitando uma maior entrada de radiação, ocorrendo alteração nas variáveis microclimáticas que não foi o caso do local experimento. Na contrapartida, o ambiente a campo por possuir uma vegetação que oferece uma proteção relativamente menor do solo, apresenta uma maior resposta ao aumento da incidência de radiação solar observada no verão. Nesta condição, com maior armazenamento de energia durante o dia, terá mais radiação térmica (terrestre) para emitir a noite.

Para Hardwick et al. (2015), a relação entre a estrutura da vegetação e o microclima pode ser explicado pelo dossel absorver, transmitir e refletir a radiação solar, portanto reduz a quantidade de energia que penetra ao longo do dossel, o que pode manter o ar e o solo abaixo do dossel em temperaturas mais baixas. Nas copas das árvores atenua a velocidade do vento na medida em que se ganha em profundidade no dossel. A turbulência do ar é suprimida pela vegetação e dosséis densos permitem menos turbulência do que dosséis mais abertos. Como o ar no topo do dossel é mais quente, a maior turbulência carrega este ar aquecido até próximo ao solo aumentando a temperatura.

A dispersão nos registros da UR, especialmente durante o dia, também surpreende, tendo variado a campo, mais da metade ao observado na floresta (Tabela 1d). No inverno, apenas na temperatura do ponto de orvalho observou-se variação significativa nos registros, sendo maior a campo $\left(16,85{ }^{\circ} \mathrm{C}\right)$ do que na floresta $\left(9,91{ }^{\circ} \mathrm{C}\right)$, durante o dia (Tabela 1e).

Hardwick et al. (2015) destacam que a quantidade de vapor de água que o ar pode manter é rigorosamente dependente da temperatura do ar. Dessa forma, em dois ambientes com a mesma umidade específica (massa de vapor de água por unidade de massa de ar), mas com diferentes temperaturas do ar, o ambiente mais quente apresentará menor umidade relativa do que o ambiente mais frio.

Para Brown (1993), as alterações que ocorrem no microclima nas diferentes vegetações, estão relacionadas principalmente com a condução da luz que atinge a clareira, onde observa-se o aumento da temperatura do ar, o aumento da umidade do solo, o aumento da evapotranspiração e a diminuição da umidade relativa do ar.
$\mathrm{Na}$ Tabela 2, estão destacados os resultados das análises de regressões, onde retrata a correlação entre os dados à campo e a floresta. A temperatura do ar (média, mínima e máxima) e do ponto de orvalho foram as variáveis de maior correlação, com valores acima de 0,92 . Os coeficientes de determinação indicam que nessas situações as variáveis obtidas a campo explicaram mais de $85 \%$ da variação na floresta. Assim, com as equações de regressão pode-se estimar a temperatura do ar e do ponto de orvalho a floresta, a partir de dados provindos de uma estação meteorológica. Apesar da UR ter tido na maioria das análises, correlações significativas, estas tiveram correlações baixas, como para o inverno (Tabela 2). Os sinais positivos dos coeficientes de correlação indicam uma relação uniformemente proporcional, isto é, a medida que uma variável a campo aumenta, a referida variável na floresta também aumenta ou vice-versa.

Tabela 2 - Coeficientes de correlação (r) e de determinação $\left(\mathrm{R}^{2}\right)$ e equação da regressão linear entre a temperatura do ar, umidade relativa e temperatura do ponto de orvalho a campo a floresta nas diferentes escalas de tempo no verão e inverno e sem distinção

\begin{tabular}{|c|c|c|c|}
\hline & $\mathrm{R}^{2}$ & $\mathrm{r}$ & Equação da Regressão \\
\hline & \multicolumn{3}{|c|}{ Temperatura média do ar } \\
\hline Geral & 0,98 & $0,98^{*}$ & $Y=0,8469 x+2,015$ \\
\hline Inverno diário & 0,98 & $0,98^{*}$ & $Y=0,9262 x+0,9773$ \\
\hline Inverno noturno & 0,98 & $0,98^{*}$ & $Y=0,8995 x+1,254$ \\
\hline Inverno diurno & 0,97 & $0,98^{*}$ & $Y=0,9629 x+0,4303$ \\
\hline Verão diário & 0,95 & $0,97^{*}$ & $Y=0,8962 x+0,8546$ \\
\hline Verão noturno & 0,90 & $0,95^{*}$ & $Y=0,8502 x+2,693$ \\
\hline \multirow[t]{2}{*}{ Verão diurno } & 0,88 & $0,93^{*}$ & $Y=0,7823 x+2,742$ \\
\hline & \multicolumn{3}{|c|}{ Temperatura mínima do ar } \\
\hline Geral & 0,98 & $0,99^{*}$ & $Y=0,8486 x+2,46$ \\
\hline Inverno & 0,95 & $0,97^{*}$ & $Y=0,8141 x+2,75$ \\
\hline \multirow[t]{2}{*}{ Verão } & 0,94 & $0,97^{*}$ & $Y=0,8537 x+2,39$ \\
\hline & \multicolumn{3}{|c|}{ Temperatura máxima do ar } \\
\hline Geral & 0,86 & $0,93^{*}$ & $Y=0,7622 x+3,642$ \\
\hline Inverno & 0,88 & $0,94^{*}$ & $Y=0,9087 x+0,8134$ \\
\hline \multirow[t]{2}{*}{ Verão } & 0,86 & $0,93^{*}$ & $Y=0,77 x+3,194$ \\
\hline & \multicolumn{3}{|c|}{ Umidade relativa do ar } \\
\hline Geral & 0,46 & $0,67^{*}$ & $Y=0,6884 x+34,62$ \\
\hline Inverno diário & 0,14 & $0,37^{* *}$ & $Y=0,4273 x+52,12$ \\
\hline Inverno noturno & 0,00 & $0,03^{\text {ns }}$ & $Y=-0,03596 x+95,17$ \\
\hline
\end{tabular}




\begin{tabular}{lccc} 
Inverno diurno & 0,35 & $0,59^{*}$ & $\mathrm{Y}=0,6343 \mathrm{x}+34,59$ \\
Verão diário & 0,73 & $0,85^{*}$ & $\mathrm{Y}=0,6741 \mathrm{x}+37,5$ \\
Verão noturno & 0,71 & $0,84^{*}$ & $\mathrm{Y}=0,5788 \mathrm{x}+47,57$ \\
Verão diurno & 0,35 & $0,59^{*}$ & $\mathrm{Y}=0,4623 \mathrm{x}+54,26$ \\
\hline \multicolumn{4}{c}{ Temperatura do ponto de orvalho } \\
\hline Geral & 0,97 & $0,98^{*}$ & $\mathrm{Y}=0,8838 \mathrm{x}+2,641$ \\
Inverno diário & 0,95 & $0,97^{*}$ & $\mathrm{Y}=0,7249 \mathrm{x}+4,283$ \\
Inverno noturno & 0,98 & $0,98^{*}$ & $\mathrm{Y}=0,7917 \mathrm{x}+2,861$ \\
Inverno diurno & 0,86 & $0,92^{*}$ & $\mathrm{Y}=0,6197 \mathrm{x}+6,464$ \\
Verão diário & 0,91 & $0,95^{*}$ & $\mathrm{Y}=0,8743 \mathrm{x}+2,936$ \\
Verão noturno & 0,85 & $0,92^{*}$ & $\mathrm{Y}=0,8431 \mathrm{x}+3,647$ \\
Verão diurno & 0,12 & $0,34^{*}$ & $\mathrm{Y}=0,3489 \mathrm{x}+12,49$ \\
\hline *Significativo a 1\%; ** Significativo a $5 \%{ }^{\text {ns }}=$ não significativo
\end{tabular}

\section{CONCLUSÕES}

Houve diferença da umidade relativa e temperatura média e máxima do ar entre ambientes a campo e em floresta densa durante o verão.

A umidade relativa do ar durante o verão apresentou maior dispersão dos dados entre os ambientes.

Pode-se estimar a temperatura do ar e do ponto de orvalho em floresta, a partir de dados obtidos de uma estação meteorológica.

\section{LITERATURA CITADA}

ALVARES, C.A.; STAPE, J.L.; SENTELHAS, P.C. et al. Köppen's climate classification map for Brazil. Meteorologische Zeitschrift, v.22, n.6, p.711-728, 2014.

ALVES, F.S.M.; FISCH, G.; VENDRAME, I.F. Modificações do microclima e regime hidrológico devido ao desmatamento na Amazônia: um estudo de caso em Rondônia (RO), Brasil. Acta Amazônica, v.29, n.3, p.395409, 1999.

BAKER, T.P.; JORDAN, G.J.; STEEL, E.A. et al. Microclimate trough space and time: Microclimate variation at the edge regeneration forest ever daily, yearly and decadal time scale. Forest Ecology Management, v.334, p.174-184, 2014.

BETTS, R.; SANDERSON, M.; WOODWARD, S. Effect of large-scale Amazon forest degradation on climate and air quality through fluxes of carbon dioxide, water, energy, mineral dust and isoprene. Philosophical Transactions of The Royal Society B, v.363, p.1873-1880, 2008.

BIUDES, M.S.; NOGUEIRA, J. de S.; DALMAGRO, H.J. MACHADO, N.G.; DANELICHEN, V.H. de M.; SOUZA, M.C. Mudança no microclima provocado pela conversão de uma floresta de Cambará em pastagem no norte do Pantanal. Revista de Ciências Agro-Ambientais, v.10, n.1, p.61-68. 2012.

BONAN, G.B. Forest and climate change: forcings, feedbacks, and the climate benefits of forests. Science, v.320, p.1444- 1449, 2009.

BROWN, N. The implications of climate and gap microclimate for seedling growth conditions in a Bornean lowland forest, Journal of Tropical Ecology, v.9, p.153168, 1993.

DAJOZ, R. Ecologia geral. 3. ed., Petrópolis, RJ: Vozes, 472p. 1978.

FISCH, G. Camada limite Amazônica: aspectos observacionais e de modelagem. Tese (Doutorado em Meteorologia). São José dos Campos, SP: INPE, 1996. 171 p.

GEISER, R. ARON, R.H.; TODHUNTER, P. The climate near the ground. 5. ed., Braunschweig, Alemanha: Vieweg, 504p. 2012.

GREISER, C.; MEINERI, E.; LUOTO, M.; EHRLIN, J.; HYLANDER, K. Monthly microclimate models in a managed boreal forest landscape. Agricultural and Forest Meteorology, v.250-251, p.147-158, 2018.

HAMMER, O.; HARPER, D.A.T.; RYAN, P.D. Past: Paleontological statistics software package for education and data analysis. Palaeontologia Electronica, v.4, n.1, p.1-9, 2001.

HARDWICK, S.R. et al. The relationship between leaf area index and microclimate in tropical forest and oil palm plantation: Forest disturbance drives change in microclimate. Agricutural and Forest Meteorology, v. 201, p.187-195, 2015.

HERNANDES, J.L. Diferenças estacionais entre variáveis microclimáticas para ambientes de interior de mata, vinhedo e posto meteorológico em Jundiaí (SP). Tese (Doutorado em Agricultura Tropical e Subtropical). Campinas, SP: Instituto Agronômico, 2002. 180p.

LIMA, A.M. de. Análise microclimática no interior e fora das florestas estacionais semideciduais na área da bacia da usina hidrelétrica de Caçugo. Revista do Departamento de Geografia - USP, São Paulo, v.27, n.2, p.67-87, 2014. 
LIN, B. B. Agroforestry management as an adaptive strategy against potential microclimate extremes in coffee agriculture. Agricultural and Forest Meteorology, v.144, n.1-2, p.85-94, 2007.

OKE, T.R. Boundary layer climates. London: Willian Clowes and Sons, 1978. 372p

SALIM NETO, S.C. Microclima em diferentes fitofisionomias de floresta de altitude na mata atlântica. Tese (Doutorado em Ciências Florestais). Jerônimo Monteiro, ES: Universidade Federal do Espírito Santo, 2017. 91p.

SILVA, M.F.A. Educação ambiental e biogeografia: avaliação das condições microclimáticas no interior de fragmentos de mata atlântica em distintos graus de conservação no município de São Cristóvão, Sergipe. Sergipe: Barlavento, 2016. 2391p.

VOURLITIS, G.L.; DA ROCHA, H.R. Flux dynamics in the Cerrado and Cerrado-Forest Transition of Brazil. In: HILL, M.J.; HANAN, N.P. (eds.) Ecosystem function in Savannas: measurement and modeling at landscape to global scales. 2010. p.97-116.

WMO. Guide to the global observing system: Volume I, Global aspects. Geneva: WMO, 60p. 2013.

ZHENG, D.; CHEN, J.; SONG, B. et al. Effects of silvicultural treatments on summer forest microclimate in southeastern Missouri Ozarks. Climate Research, v.15, p.45-59, 2000.

Recebido para publicação em 28/03/2019 e aprovado em 28/08/2019. 